\title{
An unusual case of orbito-frontal rod fence stab injury with a good outcome
}

\author{
Massimo Miscusi ${ }^{1}$, Paolo Arangio ${ }^{1}$, Luca De Martino ${ }^{2}$, Fabio De-Giorgio ${ }^{3 *}$, Piero Cascone ${ }^{4}$ and Antonino Raco ${ }^{2}$
}

\begin{abstract}
Background: High-energy non-missile penetrating injuries (stab injuries) account for a small percentage of penetrating head injuries and they present a series of special features.

Case presentation: A 35-year-old man suffered orbito-frontal? and trans-cranial injuries after falling five meters from a terrace onto a rod iron fence. The removal of the metal rod was performed outside the operating room. The orbital roof was exposed and repaired through a bifrontal craniotomy and the frontal sinuses were cranialised. The orbital floor and zygoma were plated with micro-screws.

Conclusion: The patient recovered without significant complications, apart from a slight paresis of the right superior rectus; the ocular globe remained intact.

The positive outcome obtained in this very challenging case is attributable to the competency of the Neurotrauma Unit and to the use of a synergistic approach which involved the contribution of neurosurgeons, maxillo-facial surgeons, radiologists and anaesthesiologists.
\end{abstract}

Keywords: Rod, Penetrating head injury, Orbital trauma, Cranial reconstruction

\section{Background}

High-energy non-missile penetrating injuries, also known as stab injuries, account for a small percentage of penetrating head injuries. They may be caused by knives, nails, spikes, forks, scissors, and other assorted objects. Penetration most commonly occurs through the thin bones of the skull, especially through the orbital surfaces and in the squamous portion of the temporal bone. The mechanisms of neuronal and vascular injury caused by cranial stab wounds may differ from those caused by other types of head trauma. Unlike missile injuries, no concentric zone of coagulative necrosis caused by dissipated energy is present. Unlike motor vehicle accidents, no diffuse shearing injury to the brain occurs. Unless an associated hematoma or infarct is present, cerebral damage caused by stabbing is largely restricted to the wound tract. Sometimes, a narrow elongated defect, or so-called slot fracture, is produced and diagnosed when identified [1]. However, in some cases in which skull penetration is proven, no radiological abnormality can be identified. In a series of stab

\footnotetext{
* Correspondence: fabio.degiorgio@rm.unicatt.it

${ }^{3}$ Institute of Legal Medicine, Catholic University of the Sacred Heart, 00168 Rome, Italy

Full list of author information is available at the end of the article
}

wounds, de Villiers reported a mortality of $17 \%$, mostly related to vascular injury and massive intracerebral hematomas [2]. Infections can easily complicate penetrating craniocerebral injuries and patients can develop meningitis, epidural abscess, subdural empyemas, or brain abscess. Therefore, prevention and proper management of infectious complications can lead to improved outcomes in these patients.

\section{Case presentation}

We present the case of a 35-year-old man who suffered a high-energy non-missile orbito-frontal? penetrating brain injury after falling five meters from a terrace onto a rod iron fence. The patient was transported to our Emergency Department with the rod still embedded into his right orbit and skull (Figure 1A).

The GCS score on admittance was 6. The rod was removed in the Emergency Room without any radiological control; a consequent CT scan revealed that the injury involved the right orbit floor, roof and zygoma, both the frontal sinus and the frontal lobes and had caused a frontal post-traumatic acute subdural haemorrhage. The right ocular globe was intact because the "burst" fracture of the orbital compartment (a comminute fracture of the 


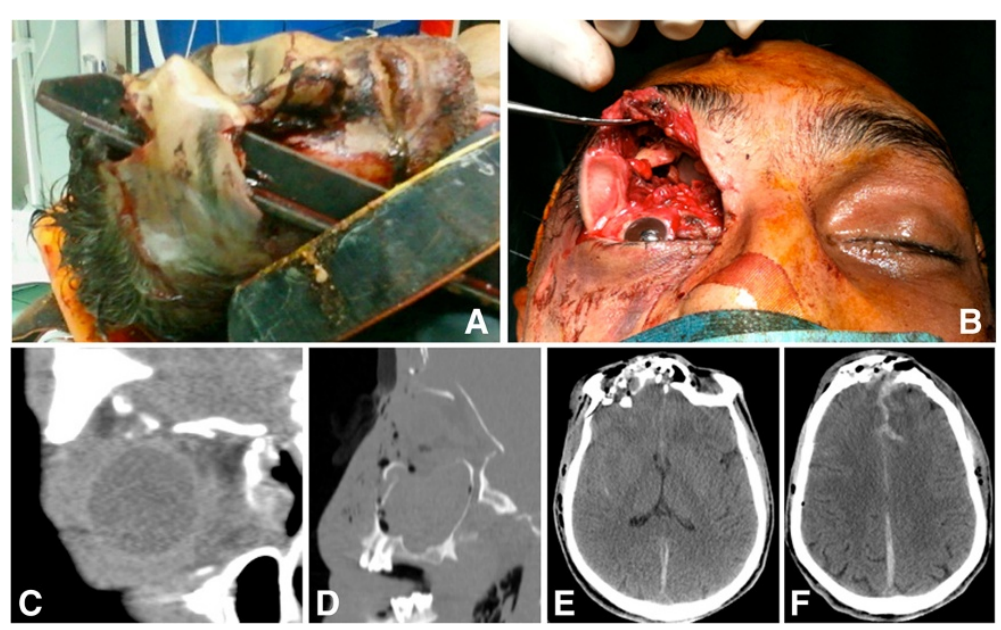

Figure 1 Patient at emergency department. A: Patient at our Emergency Department with the metal rod still embedded into the right orbit and skull. B: Inspection of the right orbit, showing a complete destruction of orbit roof with the ocular globe pushed backwards and downwards. C and D: CT coronal and sagittal scan reconstructions showing the fracture of both orbit floor and roof. E and F: $\subset T$ axial scans at admittance, demonstrating the fracture of frontal bone, with opening of frontal sinus, and signs of subarachnoid hemorrhage in the inter hemispheric cistern.

floor, roof, lateral and medial orbital walls) allowed the ocular globe to undergo a downward displacement without being smashed against an osseous wall (Figure 1B-E). Cranial and maxillo-facial CT scans excluded signs of vascular damage.

According to our imaging and treatment protocols, an angiogram was not performed because, considering the location and trajectory of the foreign body, there was no evidence of possible vascular injury. The patient immediately underwent surgery to evacuate the subdural haemorrhage and to repair both cranial and facial defects. Neurosurgeons and maxillo-facial surgeons participated simultaneously in the procedure. A bifrontal craniotomy was performed through a bicoronal flap. Intracranially, the dura mater and both frontal lobes with the first third of the superior longitudinal sinus were lacerated (Figure 2A). Haemorrhagic and necrotic tissue was debrided and the superior longitudinal sinus was clipped without difficulty. Rust was found inside the parenchyma and was removed together with (through??) a meticulous irrigation of the operative field. The frontal sinuses were cranialised, the mucosa was removed from the nasofrontal ducts. After cranialization, the nasofrontal ducts were obliterated using the temporalis muscle flap, fibrin sealant was applied over the ducts, and the pericranial flap appropriately folded in order to obliterate the dead space of the sinus. A large-scale duroplasty was performed with a bovine pericardial patch (Figure 2B). A right eyelid incision was performed to expose and reduce margin fractures using a titanium plate and screws (Figure 2C-E). A collagen membrane was used for orbital floor and roof reconstruction.
The patient was then moved to the intensive care unit and his neurological status showed a progressive and constant improvement; after fifteen days, sedation was stopped and the patient was discharged from the intensive care unit.

A large spectrum antimicrobial therapy (gram+, gram-, anaerobics and fungi) was administered continuously for one month because of the high risk of intracranial septic complications, which at the end did not occur.

A CT scan, performed one month after injury, showed a good reconstruction of the cranial vault, of the orbital floor and of the roof and demonstrated the chronic evolution of the frontal lobe injury (Figure 3A-B).

The patient was discharged to a rehabilitation unit in good general condition (GCS 15), without focal neurological signs. The right ocular globe was completely undamaged and visual acuity remained intact; however, a mild paresis of right superior rectus muscle and a slight ptosis were evident (Figure $3 \mathrm{C}$ ). The patient's neurological status and visual acuity were unchanged at the eight-month follow-up and he went back to being a street musician. He refused to undergo neuro-psychological testing to assess the frontal abilities.

\section{Conclusions}

Penetrating stab intracranial injuries caused by metallic foreign bodies are very rare among the civilian population. Only a few cases have been reported in the literature in the last two decades, but medical reports of stab wounds of the brain date from as early as 1806 [3-10]. Penfield described the pathological features of experimental stab wounds with cannulas [11]. The literature 

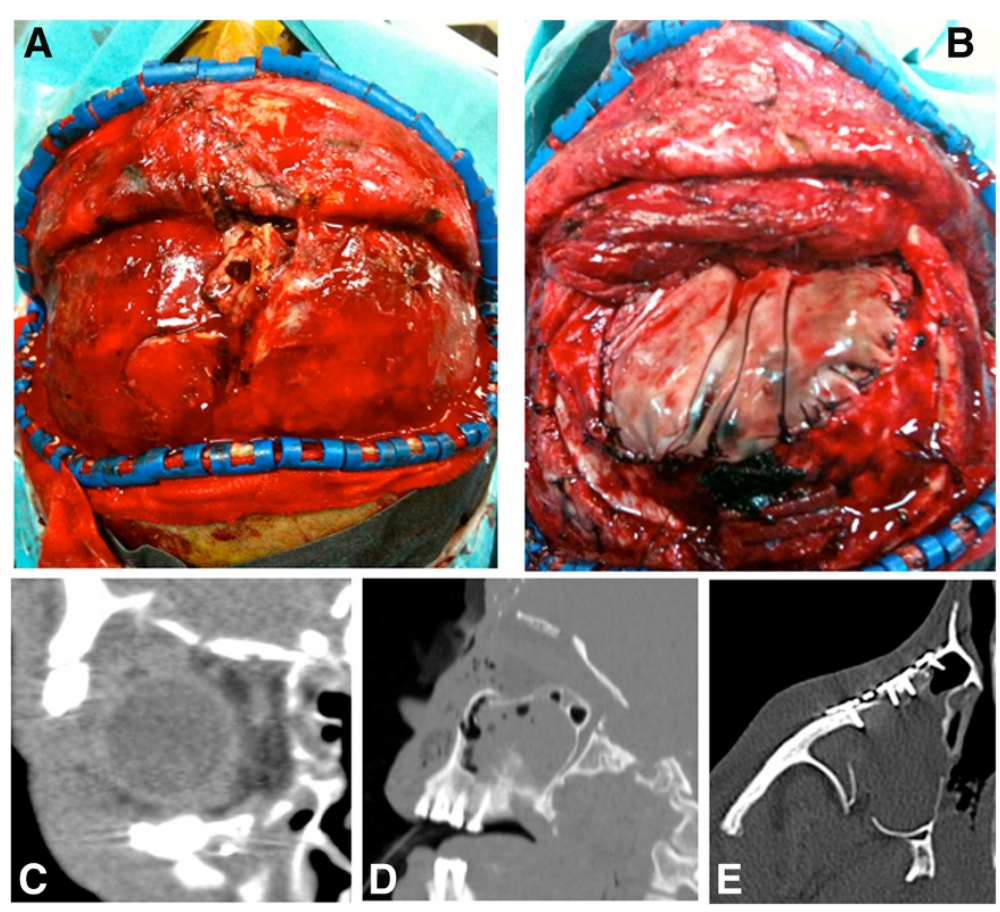

Figure 2 Skull and maxilla reconstruction: surgical and radiological view. A: Intraoperative view at the opening, with fracture of frontal bone and necrotic brain through the bone gap. B: Intraoperative view after duroplasty and cranialisation of the frontal sinus by galeal flap. C, D and E: CT scan, coronal, sagittal and axial scans, demonstrating the reconstruction of the orbit floor and roof and zygoma.

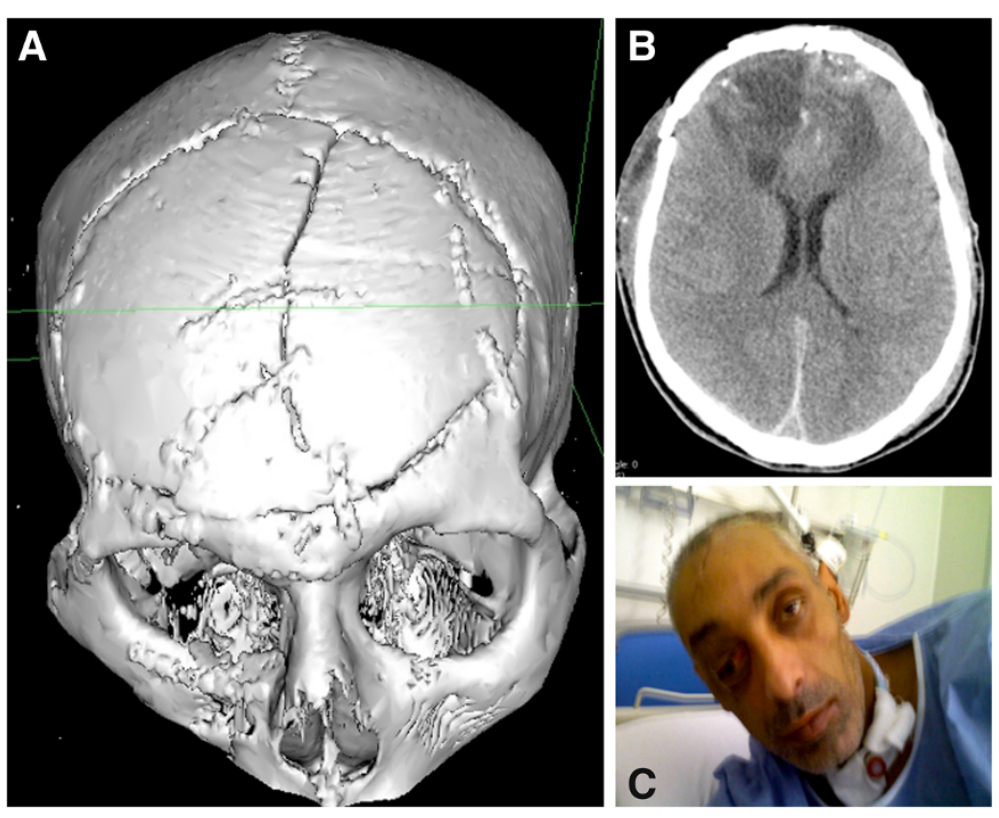

Figure 3 Clinical and radiological outcome. A: 3D CT scan view showing the final reconstruction of the fractured frontal bone. B: $C T$ scan performed one month after injury, demonstrating the hypodensity of both frontal lobes as chronic consequences of penetrating injury. C: Patient at discharge with paresis of right superior rectus muscle and ptosis. 
provides a long list of objects known to have penetrated the brain, which include knives, chopsticks, pitchforks, crochet hooks, knitting needles, brooch pins, umbrella tips, crowbars and iron rods, car antennas and scissors [12-20].

To our knowledge, this is the first report of a high-energy penetrating brain injury caused by such an object.

Penetrating craniocerebral stab injuries are more amenable to treatment than missile injuries. A stab wound creates a narrow hemorrhagic infarction which is mainly restricted to the wound tract. Concentric zones of coagulative necrosis do not result from stab wounds as they do from explosives and from the cavitating forces of missiles. Similarly, contre-coup injuries rarely occur, if at all, from stab wounds [21]. Unless the stabbing instrument is swept across the brain before withdrawal, the resultant lesion is usually focal. Therefore, when there are no direct injuries to the brain stem or direct lacerations of a major vessel, the prognosis for recovery in such type of cranial injury may be good.

Frontal stabs are accompanied by the least morbidity and mortality, while penetrating orbito-cranial stab injuries characteristically cause considerable morbidity and mortality by direct damage to brain structures, vascular compromise, or infection [22].

Vascular compromise may be due to direct vascular trauma of the internal carotid artery both in its intracavernous and paraclinoid segment, to distortion of the vessel due to local effects such as haematoma formation or oedema, or vasospasm [23].

Non-contrast cranial and maxillofacial CT scan, which is the best imaging modality for this type of trauma, can help analyze the trajectory of the foreign body and observe whether the object went through any areas of major vascular significance, including the ICA (especially within the cavernous sinus) and the anterior and middle cerebral arteries. In case of suspicion for vascular injury, an angiography should also be performed to evaluate for traumatic aneurysm, which can develop soon after a penetrating injury $[17,18]$.

In our case, in spite of the fact that the removal of the metal rod from the orbit and skull was performed outside the operating room without any radiological control, the early surgical treatment proved to be effective. Patients in whom the penetrating object is left in place until surgical removal have a significantly lower mortality than those in whom the objects are inserted and then removed (26\% versus 11\%, respectively) [24].

Blind removal of the intracranial object may cause neurological and vascular injuries if the trajectory is close to major vessels or to important neural structures. Therefore, an adequate surgical access allowing intracranial direct visualisation of the object should be performed before its withdrawal [15]. Another possibility is to remove the foreign body under CT control to enable prompt detection and management of any complications; in this case, both operating theatre and angiography should be ready [17]. In patients in whom there is no CT evidence of intracranial haemorrhage or possible damage to vascular structures, the penetrating object can be directly removed before surgical repair under general anaesthesia [18]. In our patient, even if the trajectory of the rod excluded a major vascular injury, the decision to withdraw the object blindly was made hastily because it could have been fatal for the optic nerve, compromising the visual acuity.

Surgical reparation of orbito-cranial and facial injuries was performed in a single surgical procedure by a multidisciplinary team, which included neurosurgeons and maxillo-facial surgeons.

Despite the high risk of cerebral infection due to polimicrobial contamination of intracranial compartment, our patient, who received a large spectrum antibiotics therapy, did not present any sign of meningo-encephalitis. Administration of anti-tetanus serum and antibiotics, and wound debridment by oxygen peroxide may minimise infectious complications [22].

According to the literature, antibiotic therapy should be initiated on admission and, although there are no data to support continuation of antibiotics after surgical removal, we strongly suggest to continue their administration for at least three weeks and in any case not less than one week $[17,18]$.

Furthermore, surgical interventions can achieve also a good aesthetic result, with correction of facial and cranial vault deformities. The patient presented only a slight paresis of the right superior rectus muscle due to its lacerations secondary to the passage of the rod fence through the thin orbital roof. No damage to neural structures and no sign of internal carotid involvement were demonstrated.

In conclusion, this is a unique case of penetrating orbito-cranial stab injury. Despite the removal of the rod fence from the intracranial compartment through the orbit, we obtained a very good result. The positive outcome obtained in this very challenging case is attributable to the competency of the Neurotrauma Unit and to the use of a synergistic approach. This was possible because in the Unit there is a consolidated tradition of fruitful cooperation between neurosurgeons, maxillofacial surgeons, radiologists and anaesthesiologists.

\section{Consent}

Written informed consent was obtained from the patient for publication of this Case report and any accompanying images. A copy of the written consent is available for review by the Series Editor of this journal. 


\section{Competing interests}

Authors declare that they have no competing interests.

\section{Authors' contributions}

MM conceived of the study and wrote the paper. PA performed the review of the literature and helped to write the paper. LDM helped to write the paper and to design the manuscript. FDG helped to review the literature and to draft the manuscript. PC helped to write the paper and to design the manuscript. AR participated in the coordination of the study. All authors read and approved the final manuscript.

\section{Author details}

${ }^{1}$ Department of Medical and Surgical Sciences and Biotechnologies, Rome, Italy. ${ }^{2}$ NESMOS Department, Rome, Italy. ${ }^{3}$ Institute of Legal Medicine, Catholic University of the Sacred Heart, 00168 Rome, Italy. ${ }^{4}$ Department of Odontostomatologic and Maxillo-facial Sciences, Sapienza University of Rome, Rome, Italy.

Received: 5 August 2012 Accepted: 26 June 2013

Published: 13 August 2013

\section{References}

1. Bozzeto-Ambrosi P, Costa LF, Azevedo-Filho H: Penetrating screwdriver wound to the head. Arq Neuropsiquiatr 2008:93-95.

2. De Villiers JC: Sixteen cases of transorbital stab wounds of the head. J Neurol Neurosurg Psychiatry 1975, 38(8):822.

3. Di Roio C, Jourdan C, Mottolese C, Convert J, Artru F: Craniocerebral injury resulting from transorbital stick penetration in children. Childs Nerv Syst 2000, 16(8):503-506.

4. Domenicucci M, Qasho R, Ciappetta P, Vangelista T, Delfini R: Surgical treatment of penetrating orbito-cranial injuries. Case report. J Neurosurg Sci 1999, 43(3):229-234.

5. Karim T, Topno M: An unusual case of penetrating head injury in a child. J Emerg Trauma Shock 2010, 3(2):197-198.

6. Okunaga T, Izumo T, Yoshioka T, Shimizu T, Yamashita H, Yokoyama H: A case of transorbital penetrating brain injury by a blunt metal rod. No Shinkei Geka 2010, 38(3):293-298.

7. Pascual JM, Navas M, Carrasco R: Penetrating ballistic-like frontal brain injury caused by a metallic rod. Acta Neurochir 2009, 151(6):689-691.

8. Regev E, Constantini S, Pomeranz S, Sela M, Shalit M: Penetrating craniocerebral injury caused by a metal rod: an unusual case report. Injury 1990, 21(6):414-415.

9. Stone $J$, Rifai MH, Moody RA: An unusual case of penetrating head injury with excellent recovery. Surg Neurol 1981, 15(5):369-371.

10. Mason F: Case of a young man who had a pitchfork driven into his head four inches who speedily got well. Mar 10, 1806. Lancet 1870, 1:700-701.

11. Penfield W, Buckley R: Punctures of the brain. Arch Neurol Psychiat 1928, 20:1-13.

12. Pilcher C: Penetrating wounds of the brain. Ann Surg 1936, 103:173-198.

13. Markham JW, Mccleve PE, Lynge HN: Penetrating craniocerebral injuries. J Neurosurg 1964, 21:1095-1097.

14. Dooling JA, Bell WE, Whitehurst WR: Penetrating skull wound with a pair of scissors. Case report. J Neurosurg 1967, 26:636-638.

15. Gulati A, Srinivasan B, Hunter R, Flood TR: Penetrating knife injury to the frontal lobe--a case report. Ann R Coll Surg Engl 2010, 92(6):W41-W42.

16. Mitilian D, Charon B, Brunelle F, Di Rocco F: Removal of a chopstick out of the cavernous sinus, pons, and cerebellar vermis through the superior orbital fissure. Acta Neurochir 2009, 151(10):1295-1297.

17. Schreckinger M, Orringer D, Thompson BG, La Marca F, Sagher O: Transorbital penetrating injury: case series, review of the literature, and proposed management algorithm. J Neurosurg 2011, 114(1):53-61.

18. Kim SW, Youn SK, Kim JT, Cho SH, Kim YH, Hwang KT: Management of an unusual craniofacial impalement injury by a metallic foreign body. J Craniofac Surg 2012, 23(2):e140-e146.

19. Iwakura M, Kawaguchi T, Hosoda K, Shibata Y, Komatsu H, Yanagisawa A Kohmura E: Knife blade penetrating stab wound to the brain--case report. Neurol Med Chir 2005, 45(3):172-175.

20. Chibbaro S, Tacconi L: Orbito-cranial injuries caused by penetrating nonmissile foreign bodies. Experience with eighteen patients. Acta Neurochir 2006, 148(9):937-941.
21. Lindenberg R: Trauma of meninges and brain. In Pathology of the Nervous System. Volume 2. Edited by Minckler J. New York: McGraw-Hill; 1971:1721.

22. Khalil N, Elwany MN, Miller JD: Transcranial stab wounds: morbidity and medicolegal awareness. Surg Neurol 1991, 35(4):294-299.

23. De Villiers JC: Stab wounds of the brain and skull. In Handbook of clinical neurology. Vol. 1. Edited by Vinken PJ, Bruyn JW. Amsterdam: North Holland Ed; 1975:477-493.

24. De Villiers JC, Sevel D: Intracranial complications of transorbital stab wounds. Br J Ophthalmol 1975, 59(1):52-56.

doi:10.1186/1471-2482-13-31

Cite this article as: Miscusi et al: An unusual case of orbito-frontal rod fence stab injury with a good outcome. BMC Surgery 2013 13:31.

\section{Submit your next manuscript to BioMed Central and take full advantage of:}

- Convenient online submission

- Thorough peer review

- No space constraints or color figure charges

- Immediate publication on acceptance

- Inclusion in PubMed, CAS, Scopus and Google Scholar

- Research which is freely available for redistribution

Submit your manuscript at www.biomedcentral.com/submit
C) Biomed Central 